\title{
Med.Pix
}

\section{The palms and soles}

Richard P Usatine

University of California, Los Angeles

200 UCLA Medical

Plaza, Ste 220

Los Angeles, CA

90095-1628

Correspondence to:

Dr Usatine

rusatine@ucla.edu

Competing interests:

None declared

West J Med

2000;173:160-161
QUESTION: A 31-year-old homeless man comes to a free clinic, asking if you can get rid of the spots that have appeared all over his body (figure). He tells you that these spots appeared 3 days ago. The spots are not painful and they do not itch. The patient has no other clinical symptoms and feels generally well. With further questioning, he discusses his use of crack cocaine, which has contributed to his homelessness.

A physical examination reveals hyperpigmented macules on the palms, wrists, forearms, soles, and ankles. There is slight scaling on these macules.

What additional questions would you like to ask this patient? What other parts of the body would you like to examine? What tests would help you make the diagnosis? What is your differential diagnosis at this point?

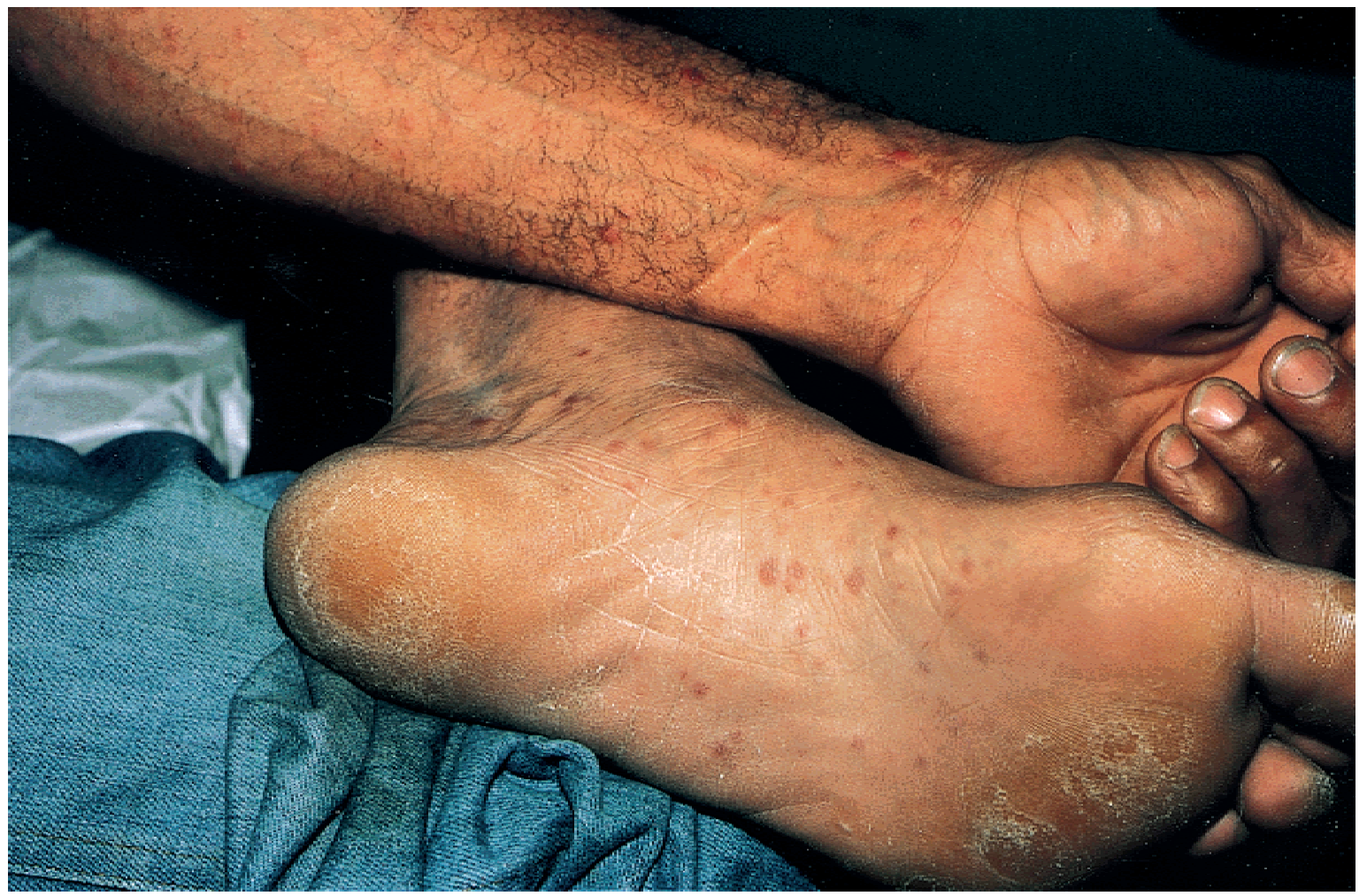

Macules on the hand and foot were not itchy or painful 
ANSWER: Taking a sexual history from the patient revealed that he had multiple female sexual partners at the time he was using crack cocaine. Some of the women provided sex for drugs. The genitals and oral mucosa were examined for additional lesions. The patient was found to have mucous patches on the penis and additional macules on the thighs. The patient also had mucous patches in the mouth.

The protean manifestations of secondary syphilis generally start 2 to 8 weeks after a chancre is present. In this case, the patient did not remember having a chancre. In secondary syphilis, $90 \%$ of patients have skin manifestations, 35\% have oral lesions, $20 \%$ have genital lesions, and $70 \%$ have constitutional symptoms, including fever and malaise. $^{1(\mathrm{p} 2479)}$

The lesions of secondary syphilis may be macular, maculopapular, papular, pustular, or annular. They are not vesicular. Macules on the palms and soles are highly suggestive of secondary syphilis. The rash is generally not itchy. About $20 \%$ of patients have mucous patches or condylomata lata. Lata, or flat, patches occur on the genitals and are flatter than condylomata acuminata. Some patients have moth-eaten alopecia.

\section{DIAGNOSIS}

The differential diagnosis included secondary syphilis, pityriasis rosea ( a benign condition that resolves over 6 to 8 weeks), and a viral exanthem. Secondary syphilis was by far the most likely diagnosis.

A VDRL test was performed. In secondary syphilis, the non-treponemal tests VDRL and rapid plasmin reagin (RPR) have a sensitivity of 99\%. A confirmatory treponemal test, such as the fluorescent treponemal antibody absorption (FTA-ABS) test, is needed to confirm a positive VDRL or RPR. As recommended by the Centers for Disease Control and Prevention, the patient was tested for HIV with his consent. ${ }^{2}$

\section{TREATMENT}

Treatment was recommended that day, even before the result of the VDRL, because of the high likelihood of secondary syphilis. The patient received an injection of 2.4 million units of penicillin $G$ in the buttocks, the recommended dose for an adequate cure of pri- mary and secondary syphilis in immunocompetent patients. $^{2}$

Penicillin is the most effective therapy for all stages of syphilis. ${ }^{2}$ Doxycycline hyclate or tetracycline are alternative treatments for patients with a demonstrated allergy to penicillin. Pregnant patients who are allergic to penicillin should be desensitized, if necessary, and treated with penicillin. ${ }^{2}$

The patient, who had no history of penicillin allergy, was observed for 1 hour in the clinic to ensure that he did not have an allergic reaction to the drug. Jarisch-Herxheimer reaction occurs in up to $90 \%$ of patients with secondary syphilis who are treated with penicillin. ${ }^{1(\mathbf{p} 2488)}$ The reaction occurs as soon as 1 to 2 hours after treatment as a result of antigen release from Treponema pallidum when the spirochetes are killed by the drug. Patients may experience chills, fever, myalgias, headache, tachycardia, hyperventilation, flushing, and mild hypotension for 12 to 24 hours. This reaction is more common in patients with secondary syphilis than in individuals with other forms of the disease, because the secondary form involves the highest number of spirochetes. The reaction is treated with antipyretic medication and rest.

The patient was counseled about safe sexual practices and offered help to stop his crack cocaine use.

\section{OUTCOME}

The VDRL test was strongly positive at a titer of 1:512, with a confirmatory positive FTA-ABS test. The patient did not return for a follow-up evaluation, which validated the importance of the decision to administer treatment on the basis of the appearance of the rash. The HIV test was negative. The positive VDRL was reported to the health department for contact tracing and treatment, which proved difficult because the patient was homeless.

References

1 Mandell GL, Bennett JE, Dolin R, eds. Principles and Practice of Infectious Diseases. 5th ed. Philadelphia: Churchill-Livingstone, 2000 .

2 Centers for Disease Control and Prevention. 1998 Guidelines for treatment of sexually transmitted diseases. MMWR Morb Mortal Wkly Rep January 23, 1998;47 (No RR-1).

Face-to-face advice to patients can help Doctors who feel they are wasting their breath giving lifestyle advice to patients should take heart. Research from St Louis suggests that face-to-face advice can prime patients to take notice of health promotion material that would otherwise be ignored or instantly forgotten (Arch Fam Med 2000;9:426-433). 